\title{
Revistas electrónicas y documentación educativa en la web
}

\author{
M. Esther del Moral Pérez \\ Departamento de Ciencias de la Educación \\ Universidad de Oviedo
}

\subsection{Resumen}

Se analizan la definición, implicaciones y características de las revistas electrónicas en el campo de la educación. Las revistas educativas en la web facilitan el acceso - tanto en velocidad como en amplitud- al conocimiento científico en el área, superando las limitaciones de las tradicionales en papel.

Palabras clave: Revistas electrónicas. Publicaciones en línea. Diseño hipermedia.

\subsection{Abstract}

The definition, implications and characteristics of online journals in the field of education are analysed. Educational web journals increase the access - both in time and extent - to scientific knowledge in the field breaking the limitations of traditional paper ones.

Keywords: Electronic magazines. Online publications. Hypermedia design.

\section{Introducción: Publicar en la red hoy}

Nos encontramos en un momento en el que se están generalizando cada vez más las publicaciones en la web. Todo tipo de empresas e instituciones se promocionan a través de páginas web muy sofisticadas, que les sirven para darse a conocer y propiciar la captación y fidelización de potenciales clientes o usuarios.

Del diseño de páginas web se ha evolucionado a la creación de portales temáticos en donde se pueden implementar todo tipo de aplicaciones, entre las que hay que mencionar las revistas electrónicas. Así pues, se trata de una práctica cada vez más común, que requiere de fórmulas específicas para su validación.

Implica unas ventajas considerables, por un lado estas publicaciones digitales suponen un bajo coste, dado que se reduce el gasto económico que conlleva el papel, el material fotográfico, etc. Y por otro, garantizan un margen de distribución 
mucho más amplio, puesto que el acceso a la misma en ocasiones desborda las expectativas, propiciando una mayor accesibilidad, a diferencia de otra clase de publicaciones de una distribución limitada por el número de ejemplares editados.

Además, poseen otra peculiaridad, y es las revistas electrónicas - por su formato de acceso y la facilidad que presentan para la participación en línea a través de los distintos servicios de comunicación que ofrece la red (correo electrónico, chats...) - generan una alta conectividad que permite un intercambio más fluido entre autores-lectores que el que hasta ahora podría derivarse de una publicación convencional.

También es preciso señalar como característica de las revistas digitales su permanente actualización de recursos, los datos obsoletos pueden modificarse fácilmente, sin que ese cambio exija un esfuerzo suplementario ni un coste adicional.

\section{Revistas en soporte digital: Revistas electrónicas}

Como ya se ha señalado, la red Internet es la artífice de la ruptura de las fronteras naturales establecidas por las limitaciones inherentes a la imposibilidad de difundir trabajos científicos en el formato tradicional de las revistas en papel.

Alfonso López Yepes (1999, p. 13) las define como el "instrumento por excelencia para la recepción, consulta, producción y difusión de información de todo tipo", y señala cinco categorías: revistas y boletines de asociaciones profesionales, publicaciones elaboradas por bibliotecas o centros de documentación, ediciones periódicas de universidades o centros de formación, revistas de instituciones de ámbitos documentales, y boletines de empresas del mundo de la información.

Todas las opiniones parecen coincidir al manifestar que se trata de aplicaciones que ya contribuyen a la divulgación del saber científico a través de la web, y que poseen un alto potencial como vehículos de comunicación e intercambio para el desarrollo de la comunidad científica.

Por otro lado, para Olmeda (1999, p. 373) la edición y la comunicación científica electrónica se constituyen en un nuevo medio que "afecta directamente a los procesos sociales implícitos en la producción del conocimiento, a los procesos sociales de formación y difusión del conocimiento experto y a la interacción entre los científicos y su medio ambiente intelectual".

La enorme potencialidad — todavía hoy no explotada en su totalidad— de estos nuevos formatos de publicación digital radica en:

- La versatilidad de los mismos;

- La rapidez de la divulgación de sus contenidos;

- El acceso a fuentes que permiten la distribución de datos relativos a estudios recientes;

Scire. $7: 2$ (jul.-dic. 2001) 85-97. 
- La posibilidad de poner en comunicación a investigadores de áreas e intereses afines.

- La capacidad de ilustrar la información adjuntando recursos multimedia.

- La narrativa hipermedia, aspecto al que dedicaremos el siguiente apartado.

\section{Narrativa hipermedia}

La narrativa, tradicionalmente, se ha ajustado a una estructura que reproduce un esquema lineal y adscrito a los cánones establecidos de un discurso unidireccional predeterminado por el autor, y cuyo itinerario era perfectamente previsible. Sin embargo, las posibilidades ofrecidas por las nuevas tecnologías y la libertad de movimiento que los hipertextos implican, han hecho posible que esa linealidad se torne en una multiplicidad de vías de lectura e interpretación.

Los hipertextos posibilitan la navegación a partir de los enlaces que se establecen entre distintos textos, términos, o palabras que implican el acceso a aclaraciones semánticas, notas complementarias, información más exhaustiva, etc., con el simple gesto de pinchar sobre un hiperenlace.

Pues bien, si a la potencialidad del hipertexto se le añade la riqueza expresiva de los recursos multimedia, obtendremos el hipermedia, capaz de presentar simultáneamente la información en los distintos formatos (imágenes reales, vídeoclips, música, animaciones...) (Del Moral, 1998, p. 190), junto con la posibilidad de formular cuestiones en forma de texto escrito y/o mediante gráficos, y de navegar libremente a través de ese volumen de información multiformato, etc. El hipermedia constituye una oportunidad para fomentar el desarrollo cognitivo de los sujetos, implicándoles directamente mediante el ejercicio continuo de toma de decisiones (Del Moral, 1999a, p. 24).

A pesar de la libertad de navegación inherente, la narrativa hipermedia no es algo fortuito, ni responde a criterios arbitrarios; mas bien todo lo contrario, ya que implica una alta estructuración del discurso contemplando fórmulas alternativas que pueden cambiar el sentido inicial del mismo, suscitando múltiples historias con entidad propia, sin que por ello parezca un compendio informe y desestructurado a modo de collage. De ahí, que sea preciso subrayar que en la narrativa hipermedia convergen tanto procesos heurísticos, morfológicos y taxonómicos, como analíticos y de lectura de la narratividad.

El hecho de que la hipermedia sea un producto de la integración coherente y estructurada de diferentes elementos expresivos, procedentes de distintos media (imagen visual, auditiva y tipográfica), hace que dé origen a historias "multitéticas interrelacionadas" que adquieren significado cuando el lector-autor recrea la obra en función de sus propias elecciones.

Scire. $7: 2$ (jul.-dic. 2001) 85-97. 
Existen unos rasgos específicos que definen a esta nueva narrativa hipermedia, entre los que pueden destacarse los siguientes:

- Libertad en el recorrido que puede trazarse el usuario a través de diferentes direcciones y sentidos.

- Nueva reorganización de espacios de comprensión y de ámbitos espaciotemporales no predeterminados.

- Recombinación de elementos narrativos materiales y formales que constituyen el relato hipermedia.

- Relatos lectocéntricos en los que el constructor del relato es el propio lector, convertido en autor a través de sus propias decisiones al optar por determinados itinerarios textuales.

- Nuevas fórmulas para presentar y explorar la información, apelando a metáforas de fácil identificación por parte del lector/usuario (Del Moral, 1999b).

- Construcción del conocimiento de forma descentralizada, compartida, dialogada, reflexiva y plural.

- Prima la heterarquía, entendida ésta como la ausencia de una jerarquía única, unidireccional preestablecida.

- Constituye un espacio social interactivo, propiciando todo tipo de relaciones dialógicas y generando un entorno rico en oportunidades de cooperación.

\section{Formato textual versus formato hipertextual}

Es evidente que nos hallamos en una situación coyuntural en la cual está teniendo lugar el fenómeno de la migración de los tradicionales soportes de texto que caracterizaban las publicaciones desde el invento de la imprenta, a soportes más sofisticados que han integrado las ventajas de las nuevas tecnologías, dando origen a los formatos hipertextuales, tan propios de la web, en donde la mayoría de lo que se publica se encuentra hiperenlazado, estableciendo infinitas relaciones con nuevas páginas web que impiden que la lectura se agote. Otro tema muy diferente sería el relativo a la coherencia y pertinencia de dichos enlaces, así como en qué medida pueden contribuir al alejamiento del interés inicial manifestado por el usuario al acceder a determinado sitio web y acabar en otros totalmente ajenos a su objetivo.

Pero, centrándo ya la atención en las revistas electrónicas — también denominadas "e-zine" en el mundo anglosajón — hay que poner de manifiesto que las nuevas tecnologías dotan a las revistas electrónicas de unas particularidades diferentes a las convencionales de papel, a saber: 
- La espacialidad de los entornos digitales,que posibilita la plena libertar de navegación y la reversibilidad de las acciones ejecutadas por el usuario (avance y retroceso sujeto a su elección).

- Disposición de un material ingente y reestructurable, propiciador de reinterpretaciones sucesivas, enriquecedoras y alternativas, que afectan a la semántica y a la hermenéutica.

- La digitalización, gracias a la cual se logra una verdadera hibridación entre los distintos recursos que pueden integrarse.

- Los textos escritos son "explotados" a través del diseño generando expresiones estéticas significativas, originales y semánticas, reproduciendo entornos metafóricos que facilitan el acceso a la información y el desplazamiento por ella.

En la figura 1 se sistematizan las diferencias más destacables entre las revistas en formato papel, y las revistas electrónicas.

\begin{tabular}{|l|l|}
\hline Revista en papel & Revista electrónica \\
\hline $\begin{array}{l}\text { Construcciones lineales predeterminadas por los } \\
\text { autores. }\end{array}$ & $\begin{array}{l}\text { Presentan hiperenlaces, con la capacidad de establecer } \\
\text { vínculos hipertextuales que facilitan el acceso a } \\
\text { documentos relacionados entre sí, algo impensable en } \\
\text { las publicaciones periódicas convencionales. }\end{array}$ \\
\hline $\begin{array}{l}\text { La publicación periódica clásica implica una sucesión } \\
\text { de volúmenes }\end{array}$ & $\begin{array}{l}\text { El lector puede elegir libremente la secuencia de } \\
\text { navegación por los contenidos de la misma. }\end{array}$ \\
\hline $\begin{array}{l}\text { Proceso de publicación más lento, sometido a } \\
\text { múltiples factores de ralentización. }\end{array}$ & $\begin{array}{l}\text { Ágil proceso de implementación y edición de los } \\
\text { contenidos en la web. En ocasiones, suele ser la versión } \\
\text { electrónica de la de papel. }\end{array}$ \\
\hline $\begin{array}{l}\text { El documento publicado ya no puede someterse a } \\
\text { nuevas revisiones. }\end{array}$ & $\begin{array}{l}\text { Abierta a posibles modificaciones, ampliaciones y } \\
\text { actualizaciones. }\end{array}$ \\
\hline $\begin{array}{l}\text { Limitación en la inclusión de ilustraciones y } \\
\text { fotografías, dado el coste suplementario de éstas. }\end{array}$ & $\begin{array}{l}\text { Incluyen prestaciones audiovisuales (combinando } \\
\text { imagen y sonido) que enriquecen los contenidos } \\
\text { textuales, sin que suponga incrementar el presupuesto. }\end{array}$ \\
\hline $\begin{array}{l}\text { Dirigidas a un público determinado con tiradas } \\
\text { limitadas que garanticen su distribución y venta. }\end{array}$ & $\begin{array}{l}\text { Logran que personas que están en lugares lejanos } \\
\text { sientan que forman parte de una comunidad, que } \\
\text { comparte los mismos intereses y objetivos. Su coste de } \\
\text { producción es mínimo y la distribución (acceso) puede } \\
\text { desbordar expectativas. }\end{array}$ \\
\hline $\begin{array}{l}\text { La participación de los lectores se limita a enviar } \\
\text { cartas a la dirección, o a realizar consultas } \\
\text { puntuales. }\end{array}$ & $\begin{array}{l}\text { Los usuarios pueden participar e interaccionar } \\
\text { generando foros y debates virtuales a través de e-mail. }\end{array}$ \\
\hline $\begin{array}{l}\text { Su financiación se hace a través de suscripciones, y } \\
\text { de la venta directa de las mismas. }\end{array}$ & $\begin{array}{l}\text { A menudo son gratuitas, y suelen financiarse a través de } \\
\text { la venta de espacios publicitarios (banners, anuncios...). }\end{array}$ \\
\hline
\end{tabular}

Scire. $7: 2$ (jul.-dic. 2001) 85-97. 
Estos nuevos formatos amplían los objetivos tradicionales y cubren nuevas necesidades. Se han convertido en unos canales de información y conocimiento más ágiles y de acceso instantáneo, que permiten a través de enlaces visitar enormes bibliotecas virtuales, consideradas como macrobases de datos temáticas.

Por otro lado, estas aplicaciones electrónicas pueden desempeñar el papel de valiosos vehículos de desarrollo de proyectos de cooperación entre distintas entidades e instituciones, como de hecho sucede, ya que existen revistas en línea que sirven de soporte para la actuación de numerosas ONGs.

Las revistas digitales se constituyen así en plataformas para la organización de actividades culturales paralelas y en soportes para la prestación de otros servicios - listas de discusión internas entre autores/lectores, chats y debates dirigidos sobre las temáticas abordadas...- explotando al máximo la capacidad de conectividad que estas revistas ofrecen (Del Moral y González Aguado, 1999).

Hasta aquí, en cierta manera, hemos estado enumerando las ventajas que este tipo de publicaciones digitales implican. Sin embargo, no es menos cierto que existen algunos inconvenientes que no se pueden obviar, y que debemos señalar, puesto que la publicación en la red tiene un componente de caos continuo:

- Se constata la necesidad de una construcción de modelos de ordenación de la información a fin de lograr un adecuado nivel de utilidad.

- Se precisa de una información puntual sobre las distintas revistas electrónicas que están surgiendo en las diversas áreas del conocimiento para facilitar su acceso.

- Se requiere la existencia de un mecanismo gestor capaz de dar de alta e indexar los nuevos documentos electrónicos periódicos dentro de la web.

Además, la proliferación de este tipo de publicaciones en línea ha ocasionado que se hayan establecido ciertos requisitos legales para que adquieran una entidad reconocida legalmente, aunque no poseen el mismo reconocimiento que las otras. Recientemente, se han adoptado ciertos mecanismos de protección de la autoría o copyright de lo que en ellas aparecen. Sin embargo, y a pesar de todo, todavía existen ciertas lagunas legales que las hacen más vulnerables que las revistas convencionales de papel.

Otro de los hándicaps de este formato de revista digital es la alta mortalidad de su información, fruto de las permanentes revisiones y actualizaciones.

\section{Claves para el diseño de revistas digitales en línea}

Hasta este punto nos hemos dedicado a la definición y conceptualización de lo que son y suponen las revistas electrónicas. Sin embargo, no es suficiente enumerar los elementos que las caracterizan, es preciso dar un paso hacia delante y 
establecer los criterios que van a ser claves en el proceso de su diseño, no sólo desde una perspectiva técnica o estética, sino teniendo presentes unos criterios de carácter pedagógico y socio-cultural que, por un lado, faciliten la contextualización de los contenidos que se abordan, y por otro, se adapten al colectivo de potenciales usuarios a los que se dirigen las mismas.

Pues bien, sin olvidar uno de los principales objetivos de las revistas electrónicas temáticas, centrado en facilitar nuevas oportunidades de acceso a determinada información de carácter científico o de divulgación, se deberá:

- Definir previamente la arquitectura de las mismas, para que posibilite la organización interna de los contenidos, la cual deberá estar fundamentada epistemológicamente con objeto de proporcionar al usuario el apoyo necesario para que se lleve a cabo una navegación significativa.

- Establecer un marco de referencia que guíe al usuario y estructure su progreso, permitiéndole realizar asociaciones, profundizar en tareas o avanzar hacia otras que puedan estar relacionadas.

- Propiciar la utilización de organizadores previos que permitan estructurar las secciones y contenidos de los que conste.

- Contemplar la utilización de sumarios, mapas conceptuales y elementos para la síntesis y organización de la información.

- Incluir ayudas instruccionales para facilitar la selección, organización e integración de la información.

- Usar metáforas familiares para expresar el contenido de la información y diseñar el interfaz.

- Presentar la información utilizando múltiples y complementarios símbolos, formatos y perspectivas, que se adapten a la temática, y que ilustren las diversas posibilidades de actuación.

- Estructurar las presentaciones e interacciones para complementar los procesos cognitivos y reducir la complejidad del procesamiento de la información.

- Ofrecer de modo intuitivo distintos tipos de asistencia táctica, instruccional y procedimental.

En último término, todo ello supone apostar por una adaptación dinámica del interfaz tanto a los usuarios como a los contenidos, capaz de generar un entorno de aprendizaje significativo, interfaz que habrá de ser (Cacheiro, 1996):

- fácil de manejar,

- original, capaz de motivar y de sorprender al usuario,

- transparente, que implique claridad tanto en la presentación de la estructura interna de los contenidos, como en las ayudas y en las estrategias que utilice;

Scire. $7: 2$ (jul.-dic. 2001) 85-97. 
- ágil, dinámico,

- homogéneo, que incorpore la misma estructura o parecida a lo largo de toda la aplicación,

- versátil, capaz de adaptarse a distintos tipos de actividades dentro de la misma aplicación,

- adaptable, que permita un uso personalizado en función de los distintos tipos de usuarios,

- multimediático, integrando de información a través de distintos formatos (texto, audio, vídeo, animaciones...),

- y potenciador de la conectividad.

A continuación se matizan un poco más los aspectos que deben tenerse en cuenta al diseñar una revista digital hipermedia.

\section{1. Características relativas al usuario y sus exigencias}

- Se debe lograr que el usuario pueda realizar rápidamente las acciones, con un bajo porcentaje de errores, y una alta satisfacción.

- Es preciso saber a priori si dichos usuarios poseen el conocimiento necesario para realizar la tarea que resuelve el problema planteado, o por el contrario, se debe incluir un tutorial, a modo de ayuda, con una pormenorizada explicación.

- Evitar, ya en la planificación de la solución, los retrasos innecesarios en el acceso a la información solicitada.

- Eliminar los elementos con capacidad distractora (animaciones, flash, etc.) cuando sean innecesarios.

- Reducir el nivel de ansiedad que puede generar en el usuario, para que éste no se bloquee o pierda el interés en la realización de las actividades propuestas.

- Incluir elementos atractivos y estimulantes con el fin de animarles a terminar las diversas tareas que se les presentan.

- Evitar los errores o, al menos, si éstos se producen, facilitar una solución sencilla para subsanarlos.

\subsection{Particularidades de la aplicación en línea:}

\subsubsection{Respecto a la velocidad:}

- Disponer de distintas velocidades de ejecución en función de los usuarios (novatos y expertos).

- Incrementar la velocidad en la realización de las actividades a medida que las tareas se hagan más familiares y comprensibles para los usuarios. 


\subsubsection{Respecto al proceso de búsqueda dentro del sistema:}

- Especificar la información objetivo junto a sus restricciones y parámetros.

- Indicar las características estructurales (capítulos o secciones) del texto.

- Decidir el itinerario a seguir para llegar a la información.

- Extraer los detalles apropiados de cada destino relevante.

- Integrar los detalles extraídos y evaluar si cumplen las especificaciones y los objetivos anteriores.

- Permitir la repetición del proceso si la evaluación fuese negativa.

\section{Dualidad información-comunicación en las revistas electrónicas}

Estamos asistiendo a la consolidación de una nueva mentalidad respecto a la información, a la comunicación y al derecho que los usuarios tienen a acceder a ellas. Ahora se concibe como una necesidad básica derivada de los avances y logros de esta sociedad en la que las Tecnologías de la Información y la Comunicación lo invaden todo, convirtiendo a los usuarios en consumidores ávidos de información, que requieren de una formación orientada a la adquisición, desarrollo y potenciación de destrezas que faciliten, a su vez, la búsqueda y la gestión de la información contenida en los distintos medios. Dicha formación debe cualificar a los usuarios para que sean capaces de acceder, seleccionar, organizar y utilizar la información que precisan para su desarrollo y enriquecimiento personal y profesional, y que, en último término, redundará en la adquisición del conocimiento. Puesto que no se puede identificar información con conocimiento, éste sólo se ocasiona a nivel cognitivo por el propio sujeto que elabora, organiza y da sentido a parte de la ingente información a la que de modo sistemático nos vemos sometidos los seres humanos (Fig. 2).

De todo lo anterior se deduce que el diseño de las revistas electrónicas que posean un cierto componente educativo o formativo debn responder a unos objetivos implícitos que se orienten a facilitar en los sujetos, finalmente la adquisición del conocimiento. En este sentido, tenemos que subrayar la idea de que dichos instrumentos tecnológicos deben:

- Potenciar la indagación y la exploración desde una perspectiva propia de la teoría del aprendizaje por descubrimiento.

- Adoptar el enfoque dialógico, al estilo de una conversación guiada, poniendo la comunicación en el centro del proceso educativo o formativo para propiciar la adquisición del conocimiento por parte del sujeto.

- Presentar un entorno constructivo, capaz de generar y expresar conocimiento a través de las asociaciones significativas que pueda establecer cada sujeto. 
Por otro lado, y muy en sintonía con las aportaciones que Internet ofrece, se deberían añadir unas nuevas exigencias, relativas a la explotación de la capacidad inherente que poseen estas revistas digitales en línea para construir una comunidad virtual de personas interesadas por los mismos temas, que propicie el intercambio y el enriquecimiento mutuo dentro de la sociedad global en la que nos hallamos inmersos. Sin embargo, hay que tener en cuenta que las nuevas tecnologías no aseguran ni garantizan la comunicación efectiva, ni las conexiones técnicas crean una comunidad, sólo la facilitan.

Esa nueva tarea de garantizar la efectividad de la comunicación queda encomendada a los gestores humanos que deben velar para que sea viable y fluida, responsables de arbitrar fórmulas y estrategias comunicativas que potencien una interactividad real y efectiva.

\section{Un caso concreto: Revistas educativas infantiles en línea}

Por último, conviene realizar ciertas sugerencias relacionadas con el diseño de revistas educativas infantiles en línea, dado que distintas instituciones - centros escolares, editoriales, empresas diversas, etc.- están utilizando este soporte para generar espacios de comunicación e intercambio entre niños/as y jóvenes:

- El usuario debe percibir que la pantalla está viva, como un mundo al que asomarse: con música de fondo, personajes interpelándonos, iconos en movimiento (monigote a modo de mascota o hilo conductor), carteles en pantalla, etc.; en definitiva, diseñando un entorno lúdico que haga atractiva la navegación a través de sus espacios.

- Los elementos integrados en las mismas deben reforzar los aprendizajes concretos que se persiguen, amenizar las tareas solicitadas, se les debe facilitar pistas ante dificultades..., y, del mismo modo, se evitará que en ningún momento el usuario quede abandonado a su suerte.

- La conjugación de los recursos interactivos y la selección de metáforas que conformen el interfaz de la revista serán aspectos claves para la definición de los ambientes concretos de aprendizaje.

Muchas de estas revistas electrónicas han logrado originar una serie de comunidades virtuales integradas por jóvenes que coinciden o comparten los mismos hobbies. En ellos, los jóvenes se registran como navegantes y pueden compartir archivos (musicales, fotográficos...) con otros miembros de la comunidad, generando un álbum de fotos virtual de cada uno de ellos. De esta forma la revista electrónica posee una doble funcionalidad: presentar artículos de divulgación (ciencia, historia, cine, arte...) y compartir un espacio privado con ciberamigos, que para muchos se ha convertido en un "barrio virtual". 
Pero, para que el resultado de esos dos sumandos de información y comunicación propicie realmente un entorno de aprendizaje se necesita algo más, a saber, un equipo de recursos humanos que mantenga, alimente y contribuya al crecimiento de la revista. Nos encontramos con un soporte que implica algo más que la mera transmisión de información, por lo que no puede quedar abocada al devenir y al dejar hacer de los usuarios. Una revista electrónica es algo vivo que, si no se nutre, muere; por lo que no es suficiente con "colgar" artículos de más o menos valor educativo o formativo sino que hay que alimentar los mecanismos de comunicación:

- Contribuyendo al refuerzo de mensajes coherentes.

- Respondiendo a las solicitudes de los usuarios en cada momento.

- Evitando excesivas actividades que impliquen poca participación (lectura en pantalla, visionado de documentos largos...).

- Fomentando la participación activa y continua de los usuarios.

- Posibilitando la conexión con otros usuarios (chat, e-mail, vídeoconferencia).

- En ocasiones, puede ser positivo registrar las decisiones del usuario y su trayectoria a través de las actividades contempladas en la revista.

Finalmente, se sugieren algunas páginas web que pueden servir como ejemplos de lo que aquí se ha abordado:

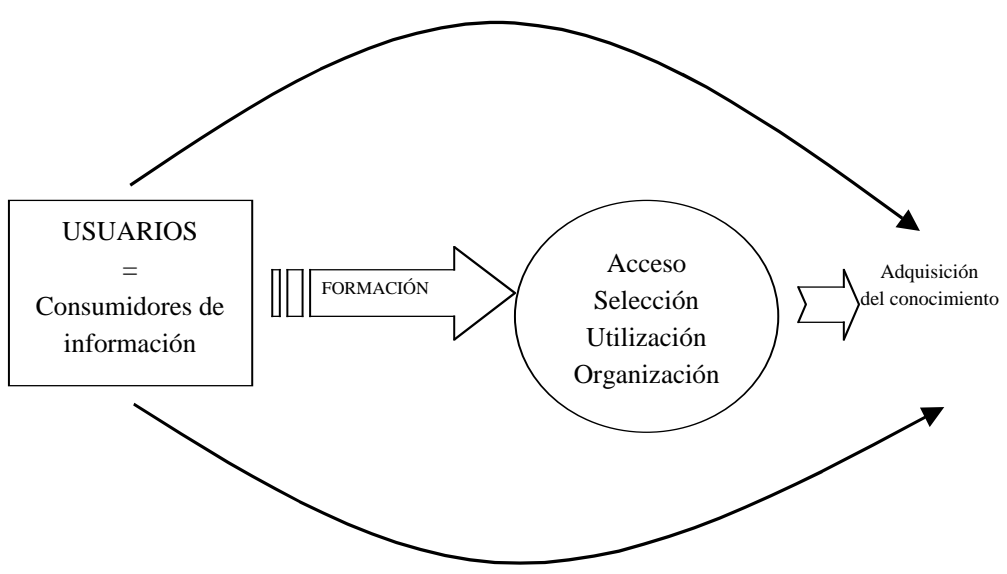

DESTREZAS DE BÚSQUEDA Y GESTIÓN DE LA

INFORMACIÓN

Figura 2. Destrezas de búsqueda y gestión de la información.

Scire. $7: 2$ (jul.-dic. 2001) 85-97. 
- http://www.encomix.es/ milaoya/

- http://www.santillana.es/scripts/santies/adinfinitun/AdInfinitun.asp

- http://www.chicos.net.ar/

- http://www.elclubdelosduendes.cl/

- http://www.chicomania.com

- http://www.comunalia.es/

- http://www.guay.com/menudos/

\section{Conclusiones}

Una de las aplicaciones que hoy contribuyen a la divulgación del saber científico a través de la web son las revistas electrónicas. La potencialidad de estos nuevos formatos de publicación digital radica en la versatilidad de los mismos; la rapidez de la divulgación de sus contenidos; la actualidad de la información; y su capacidad de poner en comunicación a investigadores de áreas e intereses afines. La red Internet es la artífice de la ruptura de las fronteras naturales establecidas por las limitaciones inherentes a la imposibilidad de difundir trabajos científicos en el formato tradicional de las revistas de papel. Debemos ser conscientes del potencial de estos vehículos de comunicación e intercambio que suponen las revistas electrónicas para el desarrollo de la comunidad científica.

La proliferación de este tipo de publicaciones en línea ha ocasionado que se hayan establecido ciertos requisitos legales para que adquieran una entidad reconocida legalmente, además de contar con los mecanismos de protección de la autoría o copyright de lo que en ellas aparecen.

Las publicaciones convencionales existentes sobre temáticas científico-culturales, y en concreto, las educativas — que serán sobre las que abundemos en el presente estudio-, se están marcando nuevos objetivos, sin dejar al margen su finalidad prioritaria en tanto que canales de información, constituyéndose en vehículos de desarrollo de proyectos de cooperación entre distintas entidades e instituciones, y plataformas para la organización de actividades culturales paralelas e incluso soportes para la prestación de servicios, aprovechando la versatilidad que presentan otros servicios de la red Internet, tales como las listas de discusión internas entre autores y lectores de dichas revistas; los chats y debates dirigidos a propósito de las temáticas abordadas, etc.; $y$, en defintiva, explotando al máximo la capacidad de conectividad que estas revistas digitales ofrecen.

Otra de las características que definen a este tipo de publicaciones electrónicas es la interactividad que consiguen a través de distintas fórmulas, algunas ya mencionadas, que - junto a una arquitectura gráfica especial que posibilita la navegación a través de la bibliografía, los glosarios y la documentación anexa y

Scire. $7: 2$ (jul.-dic. 2001) 85-97. 
mediante sofisticados motores de búsqueda - consiguen agilizar las tareas tanto de investigadores expertos en las distintas materias como de principiantes o iniciados que desean documentarse sobre algunos temas.

Sin embargo, y a pesar de todas las ventajas mencionadas, la red tiene un cierto componente caótico. Si bien, por un lado, esta característica permite una gran libertad de expresión; por otro lado, se constata la necesidad de una construcción de modelos de ordenación de la información existente a fin de lograr un adecuado nivel de utilidad, y, en este caso, la posibilidad de acceder a las distintas revistas electrónicas que están surgiendo sobre las diversas áreas del conocimiento va a depender de la existencia de un mecanismo gestor capaz de dar de alta e indexar estos nuevos documentos electrónicos periódicos dentro de la web.

Las nuevas prestaciones tecnológicas le dan a las revistas electrónicas unas particularidades que las hacen especialmente diferentes de las publicaciones convencionales de papel. En primer lugar, la publicación periódica clásica implica una sucesión de volúmenes que por las connotaciones físicas del nuevo soporte queda obviada. Además, las revistas electrónicas permiten prestaciones audiovisuales en las que se combinan imagen, sonido y texto. Otra peculiaridad que las define, es la presencia de hiperenlaces, es decir, la capacidad de establecer vínculos hipertextuales que facilitan el acceso a documentos relacionados entre sí, algo impensable en las publicaciones periódicas convencionales.

\section{Referencias}

Cacheiro González, M. L. (1996). Interfaz de navegación multimedia: diseño pedagógico. // Informática Educativa 96. Madrid : UNED, 1996., 49-157.

Del Moral Pérez, M. E. (1998). Diseños formativos de sistemas hipertextuales integrados y abiertos. // Aula Abierta. 72 (dic. 1998) 189-203.

Del Moral Pérez, M. E. (1999a). ¿Recursos multimedia en la Educación Primaria? Perspectivas ante las nuevas exigencias educativas. // Comunicación y Pedagogía. 157 (1999) 23-26.

Del Moral Pérez, M .E. (1999b). Metáforas, recursos interactivos y ambientes hipermedia para el aprendizaje. // I Jornadas de Multimedia Educativo, ICE de la Universidad de Barcelona, 5-7 julio. Barcelona: Universidad de Barcelona, 1999.

Del Moral Pérez, M. E. ; González Aguado, P. (1999). Un diseño para utilizar la red de redes para un ensayo de fortalecimiento de tejido social tan demandado en la nueva sociedad. // EDUTEC' 99. Sevilla : Universidad de Sevilla, 1999.

López Yepes, A. (1999). Innovación en la comunicación científica: las revistas electrónicas. // Scire. $5: 2$ (julio-dic. 1999) 11-21.

Olmeda Gómez, C. (1999). Revistas electrónicas y comunicación científica. // García Marco, Francisco Javier (ed.). Organización del Conocimiento en Sistemas de Información y Documentación : 3 : Actas del III Encuentro de ISKO-España. Zaragoza : Universidad de Zaragoza, 1999. 373-379.

Scire. $7: 2$ (jul.-dic. 2001) 85-97. 